\title{
When do letter features migrate? A boundary condition for feature-integration theory
}

\author{
BRIAN E. BUTLER, D. J. K. MEWHORT, and R. A. BROWSE \\ Queen's University, Kingston, Ontario, Canada
}

\begin{abstract}
Feature-integration theory postulates that a lapse of attention will allow letter features to change position and to recombine as illusory conjunctions (Treisman \& Paterson, 1984). To study such errors, we used a set of uppercase letters known to yield illusory conjunctions in each of three tasks. The first, a bar-probe task, showed whole-character mislocations but not errors based on feature migration and recombination. The second, a two-alternative forced-choice detection task, allowed subjects to focus on the presence or absence of subletter features and showed illusory conjunctions based on feature migration and recombination. The third was also a two-alternative forced-choice detection task, but we manipulated the subjects' knowledge of the shape of the stimuli: In the case-certain condition, the stimuli were always in uppercase, but in the case-uncertain condition, the stimuli could appear in either upper- or lowercase. Subjects in the case-certain condition produced illusory conjunctions based on feature recombination, whereas subjects in the case-uncertain condition did not. The results suggest that when subjects can view the stimuli as feature groups, letter features regroup as illusory conjunctions; when subjects encode the stimuli as letters, whole items may be mislocated, but subletter features are not. Thus, illusory conjunctions reflect the subject's processing strategy, rather than the architecture of the visual system.
\end{abstract}

When visual displays are presented briefly, subjects may perceive parts of the stimulus in the wrong positions as the result of spatial migration of characters or of components of characters. Such errors have figured prominently in recent analyses of early visual processing (e.g., Treisman \& Paterson, 1984), iconic memory (e.g., Mewhort, Campbell, Marchetti, \& Campbell, 1981), word recognition (e.g., Prinzmetal, Treiman, \& Rho, 1986), and lateral masking (e.g., Butler \& Currie, 1986). The present study concerns misperception arising from spatial migration of component features of letters and is based on feature-integration theory as proposed by Treisman and her colleagues (e.g., Treisman \& Gelade, 1980; Treisman, Sykes, \& Gelade, 1977).

According to feature-integration theory, features of a scene are identified preattentively-that is, in parallel, with no capacity limits. When the object to be identified is defined by a conjunction of features, the visual system must first combine the separate features into an integrated unit at a particular position. The operator responsible for feature integration is a limited-capacity spatially serial proces-

Earlier versions of these experiments were reported in a paper presented to the Canadian Psychological Association, Toronto, June 1986. The research was supported by NSERC Grant A-9581 to B. E. Butler and by NSERC Grant APA-318 to D. J. K. Mewhort. We thank Deborah Feldman-Stewart and Gregory Davel for conducting the experiments. Reprints may be obtained from Brian E. Butler, Department of Psychology, Queen's University, Kingston, Ontario, K7L 3N6, Canada. sor, named attention. In Treisman and Gelade's (1980) terms, "focal attention provides the 'glue' which integrates the initially separable features into unitary objects" (p. 98). If attention fails, features may be mismatched across objects, with the result that subjects may perceive a stimulus combination that was not actually shown. In Treisman and Paterson's (1984) words, "when attention is diverted or overloaded, simple features should be 'freefloating' with respect to one another and should therefore at times be wrongly recombined to form 'illusory conjunctions"' (p. 14).

Search experiments provide one line of support for feature-integration theory. In a typical search task, subjects must indicate, as rapidly as possible, whether or not a target is present in a display of nontarget items (foils). The target can be defined either by an individual feature or by the conjunction of several features. In the singlefeature case, feature-integration theory predicts parallel search for the target because features can be identified in parallel. For targets defined by feature conjunctions, the theory predicts serial search, whenever the foils also contain some of the target's features, because focal attention is required to fix the conjunction of features for each object.

Predictions for search tasks have been confirmed with the use of features from different dimensions and from the same dimension. Using a target defined by either color or shape, for example, Treisman, et al. (1977) found that search time was independent of the number of foils. When the target was defined by a conjunction of color and shape, 
however, they found that search time increased linearly with the number of foils (cf. Egeth, Virzi, \& Garbart, 1984). The same pattern of results was found when the target was a letter distinguished by a single feature (e.g., $R$ among $P$ and $B$ foils), rather than by a conjunction of features (e.g., $R$ among $P$ and Q; see Treisman \& Gelade, 1980).

A second line of evidence is provided by a series of ordered-report tasks, in which Treisman and Schmidt (1982) displayed rows of colored letters tachistoscopically; each row was flanked by digits, and the subjects were asked to report the digits before reporting each letter and its color. The subjects frequently mismatched the letters and colors. Treisman and Schmidt argued that, because the flanking digits had been reported first, attention was diverted away from the colored letters. As a result, the subjects were unable to integrate the features at particular locations, and features from one position migrated to another position to form illusory conjunctions. Curiously, Treisman and Schmidt did not test the simple corollary that such errors would be reduced by shifting attention to the letters.

Treisman and Paterson (1984) have argued that the evidence for feature-integration is sufficiently strong that the procedures used to test the theory can now be used to discover "the nature of the elementary units into which the sensory world is initially analyzed" (p. 12). They assume that such features can be identified by testing whether or not the features permit parallel search through a set of foils and by testing whether or not the features recombine to form illusory conjunctions (see also Treisman $\&$ Gormican, 1988). Similar arguments about perceptual units in reading have been offered by Prinzmetal and Millis-Wright (1984) and by Prinzmetal et al. (1986).

Feature-integration theory can identify elementary features only to the extent that such features remain stable across a wide range of stimuli and tasks-in Pylyshyn's (1984) terms, to the extent that they form part of the architecture of the visual system.

There are good grounds, however, to question the logic of an analysis of elementary units based on spatial migration errors. Using letter stimuli, Treisman and Gelade (1980) found that overloading attention produced spatial migration of subletter units, a result which suggests that subletter features are potential perceptual units. In contrast, Treisman and Souther (1986) found that overloading attention produced whole-character migrations within words, a result which suggests that letters are potential perceptual units. If spatial migrations are taken to define elementary units, we are lead to the contradictory conclusion that both letters and subletter units are equally elementary features. Virzi and Egeth (1984) obtained even more direct evidence that spatial migration may not be limited to elementary units. Virzi and Egeth asked subjects to report words printed in various ink colors; they found incorrect conjunctions of words and ink colors but also found that words that named colors were often reported, in error, as ink colors themselves. If we follow
Treisman and Paterson's (1984) reasoning, these results force us to conclude that word meaning is an elementary visual feature.

\section{EXPERIMENT 1}

Although errors reflecting migration of subletter units are easy to find in some tasks, we have not found them in the bar-probe partial-report experiment pioneered by Averbach and Coriell (1961). In that task, subjects are shown a multiitem display tachistoscopically and are cued to report one item from the display. Illusory conjunctions of letter features should generate errors on the basis of the similarity of the resulting feature ensemble to characters in the experiment's alphabet. If the display is small relative to the experiment's alphabet, a conjunction error is more likely to resemble a character not shown in the display than one that was shown; with, for example, a display of 8 letters selected at random from 26 , any confusion over the identity of the target should result in the subject's reporting 1 of the 18 letters that were not shown, rather than 1 of the 7 others that were shown. Quite simply, feature-integration theory predicts that extra-array errors should be more frequent- 2.6 times more common in the example cited-than intra-array errors. In contrast, Butler (1980b, 1981), Mewhort and Campbell (1978), and Mewhort et al. (1981) all reported that intra-array errors outnumber extra-array errors, often by a factor of 3 to 1 (see also Mewhort, Marchetti, Gurnsey, \& Campbell, 1984). Contrary to feature-integration theory, the majority of errors in a bar-probe task are whole-character mislocations, not errors based on misplaced letter fragments.

Why have studies with the bar-probe partial-report task yielded whole-character mislocations instead of evidence for illusory conjunctions of subletter features? One possibility, as Treisman and Souther (1986, p. 12) suggest, is the choice of stimuli; perhaps the experiments conducted to date have not been done with stimuli that encourage such errors. The first experiment, therefore, was designed to determine whether or not conjunctions of subletter features will emerge in a bar-probe task when we use stimuli known to demonstrate illusory conjunctions.

We selected our stimuli on the basis of an experiment by Treisman and Gelade (1980). They argued that focused attention is required to search for an $R$ among foils composed of $P$ and $Q$ because the cross stroke in the $R$ may shift so that subjects perceive $P$ and $Q$ instead of $R$ and $O$. The same argument applies to the letters $X$ and $Y$ (when a $Y$ is printed as an $X$ with the lower right bar omitted). Following Treisman and Gelade's analysis, then, the six letters $R, P, Q, O, X$, and $Y$ should provide suitable material for studying feature migration. Neill and Walling (1981, Experiments 3 and 4) used the letters R, P, $Q, O$ in a similar manner to test whether or not the components of a mask are confused with the features of a target.

Our task was a standard bar-probe task modified to incorporate the attention manipulation used by Treisman and 
Schmidt (1982). We presented three letters in a row flanked by a digit at each end of the row, and we manipulated attention by requiring half the subjects to attend to the flanking digits and to report them before reporting the target letters; the remaining subjects were told to ignore the digits and to report only the target letter.

The row of letters and digits was presented tachistoscopically and was followed by a mask covering all three letters and by a cue marking one letter for report (the target). The target was either a bar character $(R, Q$, and $X)$-so named because it contained a lower oblique lineor a no-bar character (P, $\mathrm{O}$, and $\mathrm{Y})$. Displays with a nobar target contained zero, one, or two nontarget bar characters. If $\mathbf{P}$ was the target, for example, the display could contain $Y$ and $O$ (no bar characters), $Q$ and $Y$ or $X$ and $O$ (one bar character), or $X$ and $Q$ (two bar characters). Likewise, displays with a bar-character target contained either zero, one, or two bar characters in addition to the target.

According to feature-integration theory, when the target is a no-bar character and the subject's attention has been diverted to the digits, extra bars in the nontarget positions should permit the subject to form an illusory conjunction and, therefore, should encourage the subject to report the character complementary to the target. As a result, accuracy for no-bar targets should decrease as the number of bar-character foils increases. Although the bar in a bar-character target may drift, there is no reason to suppose that the probability of that event is related to the presence of no-bar nontarget characters in the display. Consequently, if feature-integration theory is correct, the experiment should yield a three-factor interaction involving the attention manipulation, the type of target, and the number of bar-character nontargets.

\section{Method}

Subjects. Twenty undergraduate students participated to fulfill a course requirement in introductory psychology at Queen's University. All had normal or corrected-to-normal vision.

Materials. A total of 216 stimuli were prepared, using the bar letters $R, Q$, and $X$, the complementary no-bar letters $P, O$, and $Y$, and the digits 1-9. The form of $Y$ used in the study matched the form for $\mathrm{X}$ except for the lower right oblique bar; also, the bar on the $Q$ was exaggerated to match the bar on the $R$ and the $X$.

Each stimulus consisted of a row of five items, one digit followed by three letters and then a final digit. No items were repeated within a row.

Each letter was designated as the target for 36 trials and appeared equally often at each letter position. The remaining two letters were selected so that the target was presented along with zero, one, or two bar characters. The target's complement did not appear in the display. The positions of the additional bar items were selected at random.

The stimuli were presented on a Tektronix point-plot display monitor (Model 604) equipped with P4 phosphor and controlled by a PDP-11/23 computer. The display algorithm was based on an algorithm described by Mewhort (1978). Each letter appeared in uppercase, and all characters were formed by brightening the appropriate dots in a $13 \times 25$ matrix. A row of five characters subtended about $5^{\circ} 3^{\prime}$ of visual angle when viewed by the subject at a distance of $88 \mathrm{~cm}$. The mask character was created by brightening all dots in the matrix; the probe was a short arrow that appeared
8 ' above the target. The probe was constructed with all the dots in the "arrowhead" brightened, so that the arrow appeared as a solid triangle at the end of a straight line. Thus, neither the mask nor the probe contained the oblique bar feature that differentiated $X, Q$, and $R$ from $Y, O$, and $P$, respectively.

Procedure. At the start of each trial, a fixation dot appeared at the center of the display monitor. The subject continued the trial by pushing a button. The stimulus appeared for $50 \mathrm{msec}$ and was followed immediately by a mask covering all three letters and by the bar probe; the interstimulus interval was thus $0 \mathrm{msec}$. The mask and bar probe were shown for $75 \mathrm{msec}$. The displays were shown with a luminance of approximately $7.7 \mathrm{~cd} / \mathrm{m}^{2}$ in a small room with an ambient illumination of approximately 3.5 lux.

The subjects were told that each stimulus display would contain three letters flanked by two digits and that an arrow would indicate the letter to be reported. Subjects in the focused-attention condition were asked to ignore the digits, to report the letter indicated by the cue, and to guess if they were uncertain. Subjects in the divided-attention condition were asked to report the two digits before reporting the letter indicated by the cue and, again, to guess if they were uncertain.

Design. Each subject received 216 trials representing 12 replications for the factorial combination of three variables: (1) bar versus no-bar target, (2) position (1-3) in the row, and (3) number of bar characters (0-2) among the nontarget letters. Ten subjects were assigned to the focused-attention condition, and 10 were assigned to the divided-attention condition.

\section{Results and Discussion}

For letter report, each response was classed (1) as a correct response, if it matched the target, (2) as an intraarray error, if it matched a nontarget letter from the display, or (3) as an extra-array error. Extra-array errors were subclassified as complement errors or as intrusions. A complement error was defined as an item matching the target except for the presence or absence of the bar feature (i.e., $X$ for $Y, R$ for $P, Q$ for $O$, or vice versa); an intrusion was defined as either of the other two items that had not appeared on the particular trial.

The probability of each type of response was calculated for each subject and condition, and the scores were submitted to analyses of variance. Although display position was included as a variable in the analyses, it did not yield effects relevant to the present issues, and the data were collapsed across position. The results are summarized in Table 1.

To test feature-integration theory, we require an effective manipulation of attention. Recall that the subjects in the divided-attention condition were required to report the digits first. They reported $96 \%$ of the digits correctly, and $48 \%$ of the target letters correctly. The subjects in the focused-attention condition, by contrast, ignored the digits, and they reported $88 \%$ of the target letters correctly, which was almost twice as many as the dividedattention subjects reported $[F(1,18)=43.54, p<.0001]$. In short, the manipulation of attention was highly effective.

Feature-integration theory predicts a three-factor interaction of attention with target type and number of nontarget bar characters: When attention has been diverted, nontarget bar characters should interfere with the report of a no-bar target more than a bar-character target. As 
Table 1

Probability of Each Response Type as a Function of Target Type, the Number of Bar-Character Nontargets, and Attention in Experiment 1

\begin{tabular}{|c|c|c|c|c|c|c|}
\hline \multirow{3}{*}{$\begin{array}{l}\text { Attention } \\
\text { Condition }\end{array}$} & \multicolumn{3}{|c|}{$\begin{array}{l}\text { Bar Targets } \\
(\mathbf{R}, \mathbf{Q}, \mathbf{X})\end{array}$} & \multicolumn{3}{|c|}{$\begin{array}{l}\text { No-Bar Targets } \\
(P, O, Y)\end{array}$} \\
\hline & \multicolumn{3}{|c|}{ Number of Bar Nontargets } & \multicolumn{3}{|c|}{ Number of Bar Nontargets } \\
\hline & 0 & 1 & 2 & 0 & 1 & 2 \\
\hline \multicolumn{7}{|c|}{ Correct Responses } \\
\hline Focused & .91 & .89 & .89 & .85 & .90 & .83 \\
\hline Divided & .52 & .49 & .49 & .47 & .47 & .42 \\
\hline \multicolumn{7}{|c|}{ Intra-array Errors } \\
\hline Focused & .04 & .04 & .05 & .03 & .02 & .06 \\
\hline Divided & .28 & .35 & .32 & .28 & .29 & .33 \\
\hline \multicolumn{7}{|c|}{ Complements } \\
\hline Focused & .02 & .04 & .04 & .10 & .07 & .10 \\
\hline Divided & .06 & .07 & .09 & .12 & .14 & .14 \\
\hline \multicolumn{7}{|c|}{ Intrusions } \\
\hline Focused & .03 & .03 & .02 & .02 & .01 & .01 \\
\hline Divided & .15 & .09 & .11 & .13 & .10 & .11 \\
\hline
\end{tabular}

Table 1 shows, however, the three-factor interaction did not appear $(F<1.0)$.

Overall, accuracy for no-bar targets $(66 \%)$ was lower than for bar targets $(70 \%)[F(1,18)=3.81, .05<$ $p<.10]$. Averaging across both target types, accuracy decreased as the number of nontarget bar-characters increased $[F(2,36)=2.99, .05<p<.10]$, a trend accounted for by a relatively large difference in accuracy with one versus two nontarget bar characters $[F(1,18)=$ $8.91, p<.01]$.

Illusory conjunctions based on feature migration should be evident in the pattern of complement errors. In particular, if feature-integration theory is correct, complement errors should increase for no-bar targets as the number of bar-character nontargets increases, and the rate of increase should be larger when attention has been diverted. Instead, subjects were more likely to report the complement for a no-bar target than for a bar-character target, 0.11 compared to 0.05 , respectively $[F(1,18)=7.67$, $p<0.01]$. However, there was no evidence of interaction between target type and number of bar-character nontargets $[F(2,36)<1.0]$. Moreover, although diverting attention yielded a marginal increase in complement responses $[F(1,18)=3.62, .05<p<.10]$, there was no evidence for an interaction of attention with target type $[F(1,18)<1.0]$ or with the number of bar-character nontargets $[F(2,36)<1.0]$. Finally, there was no evidence for the three-way interaction $[F(2,36)=1.69, p>.10]$. In short, neither accuracy nor the complement errors showed the pattern of results predicted from featureintegration theory.

The main consequence of dividing attention was to increase the number of whole-character mislocations. As Table 1 shows, dividing attention increased the probability of an intra-array error from 0.04 to $0.31[F(1,18)=$ $30.43, p<.0001]$. The probability of an intrusion also increased from 0.02 to $0.11[F(1,18)=12.44, p<.01]$, but the increase was much smaller than the increase in intra-array errors.

The effects of focused-versus divided-attention became more apparent when we examined each type of error as a proportion of all errors in each condition. The proportion of complement errors - that is, responses which most resemble the actual target-dropped from $48 \%$ of all errors in the focused-attention condition to $19 \%$ in the divided-attention condition. The proportion of intrusion errors remained constant at about $20 \%$ of errors for each condition. Intra-array errors accounted for $33 \%$ of all errors in the focused-attention condition and $59 \%$ in the divided-attention condition.

Analysis of the proportion of errors suggests that, in the focused-attention condition, performance was limited because the subjects were unsure about what the target was; hence, they often had to guess, and this produced a high proportion of complement responses. In the divided-attention condition, however, the balance shifted so that intra-array responses predominated and the proportion of complement errors decreased. It appears that the burden imposed by divided attention involves information about where the target is, rather than information about the target identity. Butler $(1980 \mathrm{~b}, 1981)$ has noted the same shift in the proportion of intra- and extra-array errors as a function of the distribution of attention.

In short, dividing attention affects location information more than identity information. Although Treisman and Schmidt (1982) agree that divided attention reduces location information, they locate the loss at the feature level, where it should have a direct effect on identification (by producing illusory conjunctions). The present results, by contrast, provide evidence for whole-character mislocations without concomitant fearure migration.

The divided-attention condition required subjects both to attend to the digits and to report them first. Whenever letter report is delayed, as it was in this experiment or in the Treisman and Schmidt (1982) study, Tsal (1989, p. 396) has suggested that errors may reflect confusions in short-term memory rather than perceptual confusions.

We agree that Tsal (1989) has identified a potential difficulty, but we do not think that errors reflect memory confusion in our experiment. In a prior variation on the experiment, we asked subjects to pay attention to the digits and required half of the subjects to report the digits first and half to report the letters first. Reporting the letters first reduced the accuracy for digits from $96 \%$ to $88 \%$ but had no effect on accuracy for letters (even when we analyzed only those trials on which the digits were reported correctly). Both accuracy and the pattern of errors for letters were virtually identical to the results reported above for the divided-attention group. Hence, delaying letter report by reporting the digits first does not yield a new pattern of errors.

\section{EXPERIMENT 2}

Experiment 1 provided no evidence that divided attention results in feature migration and recombination, even 
though we used stimuli based on those used by Treisman and Gelade (1980). Treisman and Gelade, however, used a letter detection task, whereas Experiment 1 was a barprobe partial-report task. Perhaps feature migration depends on the task. To consider that possibility, a second experiment was conducted; the same displays as those in Experiment 1 were used, but the task was changed to a two-alternative forced-choice detection task.

Again, subjects were instructed either to report or to ignore the flanking digits. In addition, half of the subjects were instructed to report or to ignore the position of the target letter. Digit report was used, as before, to manipulate attention; the position report was added to determine whether or not attention to position affects letter detection. Both the feature-integration theory and Butler's (1980b, 1981) localization hypothesis suggest that knowledge of target position is provided automatically by paying attention to the target. Hence, the requirement to report position should not reduce accuracy for target report.

\section{Method}

Subjects. Four groups of six subjects each participated to fulfill a requirement in introductory psychology at Queen's University. All had normal or corrected-to-normal vision.

Materials and Apparatus. The stimuli were the same as those in Experiment 1, with the exception that the bar probe was omitted from the masking display. The apparatus was also the same. The presentation conditions were based on those in the probe task, except that the stimulus was displayed for $30 \mathrm{msec}$. After an interstimulus interval of $100 \mathrm{msec}$, the mask was presented for $1,000 \mathrm{msec}$. Thus, the stimulus onset asynchrony between target and mask was $130 \mathrm{msec}$. The luminance of the display and the illumination of the room were the same as before.

Procedure. Each subject received 216 trials presented in three blocks of 72 trials. For each block, two response alternatives were specified; the alternatives differed by one feature-the bar feature, as in $P$ and $R, O$ and $Q$, and $Y$ and $X$. Each display contained only one alternative from one of the three pairs, and both alternatives were used on half of the 72 trials in the block. The order in which the three pairs were tested was counterbalanced by blocks across subgroups of subjects.

On each trial, subjects were asked to report which of the two alternatives had appeared. In addition, half of the subjects were asked to report the flanking digits before reporting the target (the dividedattention condition), whereas the other half were instructed to ignore the digits (the focused-attention condition). Within each attention condition, half the subjects were asked to report the location of the target letter (left, middle, right), whereas half were asked to ignore position.

Design. Each subject received 12 replications for the factorial combination of three variables: bar versus no-bar target, position in the row (left, middle, or right), and number of bar nontarget letters $(0-2)$. The 24 subjects were divided into four groups: report letters only; report letter then position; report digits then letter; and report digits then letter then letter position.

\section{Results and Discussion}

The probability of reporting the digits correctly was uniformly high, about 0.98 , and was not affected by report of the position of the target $[F(1,10)<1.0]$. No further analyses of digit report were undertaken.
Letter report. The probability of reporting the target correctly was calculated for each condition and subject. The data, collapsed across letter position, are summarized in Table 2.

As shown in Table 2, the interactions obtained are precisely those predicted by feature-integration theory. Accuracy was greater for bar-character targets than for no-bar targets $[F(1,20)=6.49, p<.02]$, and it decreased as the number of bar-character nontargets increased $[F(2,40)=5.68, p<.01]$. The decrease was larger for no-bar targets than for bar targets $[F(2,40)=$ $10.48, p<.01]$. Moreover, dividing attention exaggerated the interaction of target type with number of bar nontargets, yielding a three-way interaction of target type, number of bar characters, and attention $[F(2,40)=3.39$, $p<.05]$. In short, as predicted from feature-integration theory, subjects misperceived $P, O$, and $Y$ (as $R, Q$, and $X$, respectively) when those targets appeared among other bar characters and attention was divided.

As Table 2 shows, letter accuracy was not affected by the requirement to report the target's position $[F(1,20)<1.0]$, although it was reduced substantially by reporting the digits first $[F(1,20)=12.87, p<.01]$. Position information appears to be an automatic consequence of attention to the target.

Position report. The probability of correct position report was calculated for each condition and subject. The data, collapsed across position, are summarized in Table 3.

Like letter accuracy, position accuracy decreased (from $99 \%$ in the focused-attention condition to $77 \%$ in the divided-attention condition $[F(1,10)=22.02, p<.001]$. Unlike letter accuracy, position accuracy was higher for no-bar targets than for bar-character targets $[F(1,10)=$ $11.34, p<.01$ ], and declined as the number of bar nontargets increased $[F(2,20)=5.32, p<.02]$. The decrease was greater for the bar-character targets than for the no-bar targets $[F(2,20)=4.99, p<.02]$. In contrast to the pattern in the bar-probe task, then, position accuracy was poorest when a bar-character target appeared among items that shared the bar as a common feature.

Table 2

Probability of Correct Letter Detection as a Function of Target Type, the Number of Bar-Character Nontargets, Attention, and Location Report in Experiment 2

\begin{tabular}{|c|c|c|c|c|c|c|}
\hline \multirow{3}{*}{$\begin{array}{l}\text { Attention } \\
\text { Condition }\end{array}$} & \multicolumn{3}{|c|}{$\begin{array}{l}\text { Bar Targets } \\
(\mathbf{R}, \mathbf{Q}, \mathbf{X})\end{array}$} & \multicolumn{3}{|c|}{$\begin{array}{l}\text { No-Bar Targets } \\
(P, O, Y)\end{array}$} \\
\hline & \multicolumn{3}{|c|}{ Number of Bar Nontargets } & \multicolumn{3}{|c|}{ Number of Bar Nontargets } \\
\hline & 0 & 1 & 2 & 0 & 1 & 2 \\
\hline \multicolumn{7}{|c|}{ No Location Report } \\
\hline Focused & .97 & .98 & .94 & .95 & .94 & .92 \\
\hline Divided & .87 & .86 & .87 & .88 & .85 & .79 \\
\hline \multicolumn{7}{|c|}{ Location Report } \\
\hline Focused & .95 & .91 & .95 & .94 & .94 & .89 \\
\hline Divided & .86 & .85 & .90 & .85 & .85 & .75 \\
\hline
\end{tabular}


Table 3

Probability of Correct Report of Target Position as a Function of Target Type, the Number of Bar-Character Nontargets, and Attention in Experiment 2

\begin{tabular}{|c|c|c|c|c|c|c|}
\hline \multirow{3}{*}{$\begin{array}{l}\text { Attention } \\
\text { Condition }\end{array}$} & \multicolumn{3}{|c|}{$\begin{array}{c}\text { Bar Targets } \\
(\mathrm{R}, \mathrm{Q}, \mathrm{X})\end{array}$} & \multicolumn{3}{|c|}{$\begin{array}{l}\text { No-Bar Targets } \\
(\mathrm{P}, \mathrm{O}, \mathrm{Y})\end{array}$} \\
\hline & \multicolumn{3}{|c|}{ Number of Bar Nontargets } & \multicolumn{3}{|c|}{ Number of Bar Nontargets } \\
\hline & 0 & 1 & 2 & 0 & 1 & 2 \\
\hline & 99 & 99 & .99 & 98 & 1.00 & .97 \\
\hline Divided & .81 & .71 & 68 & .81 & .82 & .80 \\
\hline
\end{tabular}

In summary, focusing attention decreased the number of illusory conjunctions and increased position accuracy. Tsal (1989) has suggested that a direct link between these two responses can be established by calculating conditional probabilities; " with such a paradigm the theory predicts that correctly conjoined features will also be correctly localized, whereas incorrectly conjoined features will largely be mislocalized" (p. 396). In principle, we could extend the present analysis and examine no-bar targets with one bar character in the display to discover whether illusory conjunctions involve mislocating the main character, the bar feature, or both; in practice, however, the probability of guessing is too high and the number of trials too few to produce a meaningful analysis with the present data set.

\section{EXPERIMENT 3}

According to feature-integration theory, illusory conjunctions occur when an elementary feature shifts position and amalgamates, precategorically, with an adjacent object. The probability of an illusory conjunction is thought to depend on how attention is focused.

In the first two studies, the same stimuli (and hence the same elementary features), the same attention manipulation, and the same criterion for scoring feature migrations were used. Nevertheless, the studies yielded quite different results. When attention was diverted, the bar-probe task produced evidence for whole-character mislocations but no evidence for feature migration; the detection task, by contrast, yielded illusory conjunctions exactly as predicted from feature-integration theory.

Illusory conjunctions must, therefore, depend on a factor not anticipated by feature-integration theory. One possibility concerns the nature of the information required in the task. The bar-probe task stresses the use of both position and identity information, whereas the detection task stresses identity only. Perhaps, the difference in stress on position information can explain the difference in results for the two tasks. The question remains open, but we think that the difference in stress is not an adequate answer. Recall that half the subjects in Experiment 2 were asked to report position information. If the difference in stress on position information were responsible for the difference in results across the two experiments, the subjects who were required to report the target's position should have shown an error pattern more similar to that in Experiment 1.

Another possibility is that the two tasks encouraged different encoding strategies and that feature migration depends on the strategy used. In Experiment 1, the target could be any one of six items, and the pattern of errors suggested that subjects treated the stimuli as whole letters. In Experiment 2, by contrast, the target was always one of two letters that differed in terms of only one feature; if the choices were $P$ or $R$, the subjects knew that $P$ had to be present so they had only to determine whether the bar was present and, if so, whether it was joined to the $P$. The pattern of errors suggests that subjects encoded the stimuli as fearure sets rather than whole letters.

Recognition accuracy is usually enhanced by reducing the number of response alternatives (Garner, 1962). With backward masking, however, providing response alternatives before the stimulus often reduces accuracy (Smith, Haviland, Reder, Brownell, \& Adams, 1976). Smith et al. suggest that presenting two choices allows subjects to adopt a strategy of looking for specific features; once they have adopted this strategy, they are unable to determine whether or not the features occurred in the target or in the mask. Neill and Walling (1981) extended the idea by showing that such disruption occurs only when the alternatives are highly similar to each other (they used $P-R$, $\mathrm{O}-\mathrm{Q}$ ) and when the mask contains the distinguishing feature. Neill $(1985$, p. 482$)$ concluded that presenting the alternatives before the display encourages subjects to encode the stimulus at a physical level, whereas presenting the alternatives after the display encourages subjects to encode at a name level.

Neill (1985) provided further evidence to support the encoding argument, by showing that the disruption effect depends on the subject's being able to predict the shape of the targets. Disruption occurred with letters when all targets were uppercase, so that the two alternatives ( $P$ and R) reliably defined a critical physical feature, but did not occur when the targets could be either upper- or lowercase, so that the physical shape was not predictable.

Experiment 3 was designed to determine whether feature migration depends on the subject's encoding strategy. A manipulation similar to that of Neill's (1985) was used; that is, the letter detection task was repeated under two uncertainty conditions. The task was the same as the divided-attention, no location-report condition in Experiment 2. For one group of subjects, the stimuli were presented in uppercase, and the shape of the alternatives was predictable, the case-certain condition. A second group, the case-uncertain condition, received precisely the same stimuli and the same instructions, but the stimuli were mixed randomly with an equal number of lowercase stimuli. The case-uncertain subjects were aware that a specific pair of letters-P or $R$, for example-formed the targets for a block of trials, but they could not predict whether the target would appear in upper- or lowercase.

If our analysis is correct, subjects in the case-certain condition should encode the stimuli as feature groups 
rather than letters; their strategy would be to look for the critical bar feature. Uncertainty about the case of the letters should force the case-uncertain subjects to encode the stimuli as letters, rather than feature groups. Consequently, subjects in the case-certain condition should show an increased error rate for no-bar targets surrounded by bar-character nontargets, whereas subjects in the caseuncertain condition should not.

\section{Method}

Subjects. Eighteen subjects participated to fulfill a requirement for a second-year psychology course at Queen's University summer session. All had normal or corrected-to-normal vision. Twelve were assigned to the case-certain condition, and 6 were assigned to the case-uncertain condition; only 6 were included in the latter condition because of the difficulty of obtaining subjects in the late summer.

Materials and Apparatus. As in Experiment 2, the 216 stimuli consisted of three letter sequences based on $P, R, O, Q, Y$, and $X$, with a flanking digit at each end of the letter row. In the casecertain condition, all 216 stimuli were in uppercase characters; in the case-uncertain condition, the stimuli on half of the trials were presented in uppercase letters and on half the trials in lowercase letters. The apparatus and presentation conditions were the same as in Experiment 2.

Procedure. Each subject received 216 trials presented in three blocks of 72 trials each. For each block, two response alternatives were specified and, as in Experiment 2, the alternatives were $P$ and $R, O$ and $Q$, or $Y$ and $X$. For the subjects in the case-uncertain condition, upper- and lowercase stimuli appeared equally often within each block, but the order of presentation was randomized so that subjects could not predict the character's case for a given trial. Again, the order of blocks was counterbalanced across subgroups of subjects. On each trial, the subjects were required to report the two flanking digits before reporting which of the two alternatives had been presented.

Design. Each subject in the case-certain condition received 12 replications for the factorial combination of three variables: bar versus no-bar target; position in the row (left, middle, or right); number of bar nontargets (0-2). Each subject in the case-uncertain condition received 6 replications for the factorial combination of each of the variables just mentioned, plus upper-versus lowercase.

\section{Results and Discussion}

The probability of reporting the digits correctly was 0.98 for the case-certain condition and 0.96 for the caseuncertain condition. We present the results only for trials on which the digits were reported correctly-that is, for the trials on which we know the subjects divided their attention. For subjects in the case-uncertain condition, the primary data concern only trials with uppercase stimuli, inasmuch as the variables of interest are relevant only for those trials. The data concerning the lowercase letters were analyzed separately.

Table 4 shows the probability of detecting the correct letter as a function of the type of target, number of nontarget bar characters, and case uncertainty. As in the preceding studies, the data have been collapsed across target position.

The case-certain condition yielded the interaction predicted by feature-integration theory, whereas the caseuncertain condition did not. Overall, the no-bar targets were more difficult to detect than the bar-character tar-
Table 4

Probability of a Correct Letter Detection as a Function of Target Type, the Number of Bar-Character Nontargets, and Case Certainty in Experiment 3

\begin{tabular}{|c|c|c|c|c|c|c|}
\hline \multirow[b]{3}{*}{ Case } & \multicolumn{3}{|c|}{$\begin{array}{c}\text { Bar Targets } \\
(\mathrm{R}, \mathrm{Q}, \mathrm{X}) \\
\end{array}$} & \multicolumn{3}{|c|}{$\begin{array}{l}\text { No-Bar Targets } \\
(P, O, Y)\end{array}$} \\
\hline & \multicolumn{3}{|c|}{ Number of Bar Nontargets } & \multicolumn{3}{|c|}{ Number of Bar Nontarget: } \\
\hline & 0 & 1 & 2 & 0 & 1 & 2 \\
\hline Certain & .84 & .84 & .85 & .81 & .76 & .65 \\
\hline Uncertain & .91 & .91 & .93 & .84 & .73 & .79 \\
\hline
\end{tabular}

Note-Data are for uppercase stimuli only and only for those trials on which the accompanying digits were reported correctly.

gets $[F(1,15)=49.52, p<.001]$, and accuracy for both targets decreased as the number of bar-character nontargets increased $[F(2,30)=10.22, p<.001]$. The decrease associated with an increased number of bar nontargets was greater for the no-bar targets than for the barcharacter targets $[F(2,30)=11.03, p<.001]$. The interaction between type of target and number of bar nontargets was greater for the case-certain condition than for the case-uncertain condition $[F(2,30)=5.37, p<.04]$. The predictability of target shape in the case-certain condition made the subjects more sensitive to illusory conjunctions.

The three-way interaction was confirmed by separate analyses for the two groups of subjects. An analysis of variance for the case-certain condition showed a substantial two-factor interaction between type of target and the number of bar nontargets $[F(2,22)=11.79, p<.001]$. A similar analysis for the case-uncertain condition showed no effect of number of bar nontargets $[F(2,10)=1.55]$, nor any evidence of an interaction of type of target with number of bar nontargets $[F(2,10)=1.6]$. Paying attention to the digits, therefore, increases the likelihood of feature regrouping, but only when the task encourages the subjects to treat the displays as collections of features rather than whole items.

The present results are quite similar to those reported by Neill (1985), who used the same letter pairs. Both his study and ours support the claim of Posner, Boies, Eichelman, and Taylor (1969), that "whether or not, under a particular condition, $S$ s choose to attend to the visual features seems to be something that $E$ can manipulate by incentive or by varying the task conditions" (p. 16).

\section{CONCLUSIONS}

In these experiments, a prediction derived from featureintegration theory was examined-namely, that the frequency of illusory conjunctions increases when attention is diverted or overloaded (Treisman \& Paterson, 1984). To test the hypothesis, we used stimuli known to yield illusory conjunctions in each of three tasks, a bar-probe task and two two-alternative forced-choice detection tasks. The bar-probe task yielded whole-character localization errors, not errors based on feature migration and recombination. The detection tasks yielded mislocalizations 
based on feature migrations, but only when subjects were permitted to treat the stimuli as feature groups rather than as postcategorical objects.

The same stimuli can yield both feature and character migrations. The unit of migration depends on the subject's encoding and selection strategy. If the subjects encode the stimuli as aggregates of features and select precategorically, feature migrations will occur. If the subjects encode the stimuli as whole characters and select postcategorically, whole-character mislocalizations will occur.

Because feature-integration theory does not specify how subjects choose their encoding and selection strategy, our results suggest that it is incomplete. For the same reason, our results argue that Treisman and Paterson (1984) were incorrect in the claim that feature-integration theory can use illusory conjunctions to derive the perceptual units "into which the sensory world is initially analyzed"' (p. 12). Their claim assumes, in Pylyshyn's (1984, p. xvi) terms, that illusory conjunctions reflect a property of the architecture of the visual system and, hence, are stable across tasks. Our results argue the contrary: Illusory conjunctions do not reflect the system's architecture; they depend on the subjects' encoding and selection strategy. In short, illusory conjunctions reflect the characteristics of the task as much as the architecture of the visual system. If stimuli are always analyzed in terms of the same elementary units, as Treisman and Paterson (1984) imply, and if those units can be trapped into revealing themselves via spatial migration errors, we still do not know how to set the subjects' strategy and, hence, how to choose a task that can focus on the correct unit. Thus, our results offer little support for the idea that feature-integration theory can drive the discovery of perceptual units.

The data pose two other puzzles for feature-integration theory. Given that letters are composed of elementary features, feature-integration theory assumes that focal attention will be needed to integrate the features into letters. As a result, the features will be glued together at one location. Once features have been glued together at a location, it follows that all spatial migrations-especially the kind of whole-character migrations we found in Experiment 1 -should be rare. Second, if features become unglued, we would expect both feature-based and wholecharacter mislocations, but not one without the other; feature-integration theory offers no way of binding features without tying them to a location.

A final point about selection deserves comment; a number of studies have shown that conceptual category (letters vs. digits) can be an effective cue for partial report (Butler, 1980a; Dick, 1970; Duncan, 1983b; Merikle, 1980; see also Mewhort \& Butler, 1983). Jonides and Gleitman (1972) demonstrated selection by category in a detection task, but others, also using a two-alternative forced-choice procedure, have failed to replicate their results (Corcoran \& Jackson, 1977; Duncan, 1983a; Krueger, 1984). The latter studies may have encouraged subjects to encode the targets as sets of features rather than whole characters; if a degree of shape uncertainty were introduced, as it was in Experiment 3, the same tasks might well show selection by category.

\section{REFERENCES}

Averbach, E., \& Coriell, A. S. (1961). Short-term memory in vision. Bell System Technical Journal, 40, 309-328.

BuTLER, B. E. (1980a). The category effect in visual search: Identification versus localization factors. Canadian Journal of Psychology, 34, 238-247.

BUTLER, B. E. (1980b). Selective attention and stimulus localization in visual perception. Canadian Journal of Psychology, 34, 119-133.

BuTLER, B. E. (1981). Identification and localization in tachistoscopic recognition: The effects of data- and resource-limitations. Canadian Journal of Psychology, 35, 36-51.

Butler, B. E., CuRrie, A. (1986). On the nature of perceptual limits in vision: A new look at lateral masking. Psychological Research, 48, 201-209.

Corcoran, D. W. J., JACKson, A. (1977). Basic processes and strategies in visual search. In S. Domic (Ed.), Attention and performance $V I$ (pp. 387-411). New York: Erlbaum.

Dick, A. O. (1970). Visual processing and the use of redundant information in tachistoscopic recognition. Canadian Journal of Psychology, 24, 133-141.

DUNCAN, J. (1983a). Category effects in visual search: A failure to replicate the "oh-zero" phenomenon. Perception \& Psychophysics, 34, 221-232.

Duncan, J. (1983b). Perceptual selection based on alphanumeric class: Evidence from partial reports. Perception \& Psychophysics, 33, 533547.

Egeth, H. E., Virzi, R. A., \& GARBART, H. (1984). Searching for conjunctively defined targets. Journal of Experimental Psychology: Human Perception \& Performance, 10, 32-39.

GARNER, W. R. (1962). Uncertainty and structure as psychological concepts. New York: Wiley.

Jonides, J., \& Gleitman, H. (1972). A conceptual category effect in visual search: $\mathrm{O}$ as letter or as digit. Perception \& Psychophysics, $12,457-460$.

KRUEGER, L. E. (1984). The category effect in visual search depends on physical rather than conceptual differences. Perception \& Psychophysics, 35, 558-564.

MenkLe, P. M. (1980). Selection from visual persistence by perceptual groups and category membership. Journal of Experimental Psychology: General, 109, 279-295.

MEWHORT, D. J. K. (1978). DIS: An n-channel tachistoscope algorithm. Behavior Research Methods \& Instrumentation, 10, 756-760.

Mewhort, D. J. K. Butler, B. E. (1983). On the nature of brief visual storage: There never was an icon. Behavioral \& Brain Sciences, 6, 31-33.

Mewhort, D. J. K., Campaell, A. J. (1978). Processing spatial information and the selective-masking effect. Perception \& Psychophysics, 24, 93-101.

Mewhort, D. J. K., Campbell, A. J., Marchetti, F. M., \& CampBELL, J. I. D. (1981). Identification, localization, and "iconic memory": An evaluation of the bar-probe task. Memory \& Cognition, 9, 50-67.

MeWhort, D. J. K., Marchetti, F. M., Gurnsey, R., \& Campbell, A. J. (1984). Information persistence: $A$ dual-buffer model for initial visual processing. In H. Bouma \& D. G. Bouwhuis (Eds.), Attention and performance $X$ : Control of language processes (pp. 287 298). London: Erlbaum.

NeILL, W. T. (1985). Levels of processing in disruptive effects of prior information. Memory \& Cognition, 13, 477-484.

NeILl, W. T., \& Walling, J. R. (1981). Disruptive effects of prior information on tachistoscopic recognition. Memory \& Cognition, 9 , 217-224.

Posner, M. I., Boies, S. J., Eichelman, W. H., \& Taylor, R. L. (1969). Retention of visual and name codes of single letters. Journal of Experimental Psychology Monograph, 79, 1-16.

Prinzmetal, W., \& Mllis-Wright, M. (1984). Cognitive and lin- 
guistic factors affect visual feature integration. Cognitive Psychology, 16, 305-340.

Prinzmetal, W., Treiman, R., Rho, S. H. (1986). How to see a reading unit. Journal of Memory \& Language, 25, 461-475.

PyLYSHYN, Z. (1984). Computation and cognition: Toward a foundation for cognitive science. Cambridge, MA: MIT Press.

Smith, E. E., Haviland, S. E., Reder, L. M., Brownell, H., \& AdAms, N. (1976). When preparation fails: Disruptive effects of prior information on perceptual recognition. Joumal of Experimental Psychology: Human Perception \& Performance, 2, 151-161.

Treisman, A. M., \& Gelade, G. (1980). A feature-integration theory of attention. Cognitive Psychology, 12, 97-136.

Treisman, A. M., \& Gormican, S. (1988). Feature analysis in early vision: Evidence from search asymmetries. Psychological Review, 95 , $15-48$

Treisman, A. M., \& Paterson, R. (1984). Emergent features, attention, and object perception. Journal of Experimental Psychology: $\mathrm{Hu}$ man Perception \& Performance, 10, 12-31.

Treisman, A. M., \& SchmidT, H. (1982). Illusory conjunctions in the perception of objects. Cognitive Psychology, 14, 107-141.
Treisman, A. M., Souther, J. (1986). Illusory words: The roles of attention and of top-down constraints in conjoining letters to form words. Jourmal of Experimental Psychology: Human Perceprion \& Performance, 12, 3-17.

Treisman, A. M., Sykes, M., Gelade, G. (1977). Selective attention and stimulus integration. In S. Dornic (Ed.), Attention and performance VI (pp. 333-361). Hillsdale, NJ: Erlbaum.

TSAL, Y. (1989). Do illusory conjunctions support the feature integration theory? A critical review of theory and findings. Journal of Experimental Psychology: Human Perception \& Performance, 15 , $394-400$.

VIRZI, R. A., \& EGETH, H. E. (1984). Is meaning implicated in illusory conjunctions? Journal of Experimental Psychology: Human Perception \& Performance, 10, 573-580.

(Manuscript received September 27, 1989; revision accepted for publication August 13, 1990.) 